\title{
Scanning Laser Topography and Scanning Laser Polarimetry: Comparing Both Imaging Methods at Same Distances from the Optic Nerve Head
}

\author{
Stephan Kremmer ${ }^{*}, 1,2$, Marcus Keienburg ${ }^{2}$, Gerasimos Anastassiou ${ }^{1,2}$, Maurice Schallenberg ${ }^{2}$, \\ Klaus-Peter Steuhl ${ }^{2}$ and J. Michael Selbach ${ }^{1,2}$ \\ ${ }^{I}$ Eye Hospital, Evangelisches Krankenhaus Gelsenkirchen, Munckelstr. 27, D-45879 Gelsenkirchen, Germany \\ ${ }^{2}$ Department of Ophthalmology, University of Duisburg-Essen, Hufelandstr. 55, D-45122 Essen, Germany
}

\begin{abstract}
Purpose: To compare the performance of scanning laser topography (SLT) and scanning laser polarimetry (SLP) on the rim of the optic nerve head and its surrounding area and thereby to evaluate whether these imaging technologies are influenced by other factors beyond the thickness of the retinal nerve fiber layer (RNFL).

Materials and Methodology: A total of 154 eyes from 5 different groups were examined: young healthy subjects (YNorm), old healthy subjects (ONorm), patients with normal tension glaucoma (NTG), patients with open-angle glaucoma and early glaucomatous damage (OAGE) and patients with open-angle glaucoma and advanced glaucomatous damage (OAGA). SLT and SLP measurements were taken. Four concentric circles were superimposed on each of the images: the first one measuring at the rim of the optic nerve head (1.0 ONHD), the next measuring at 1.25 optic nerve head diameters (ONHD), at 1.5 ONHD and at 1.75 ONHD. The aligned images were analyzed using GDx/NFA software.

Results: Both methods showed peaks of RNFL thickness in the superior and inferior segments of the ONH. The maximum thickness, registered by the SLT device was at the ONH rim where the SLP device tended to measure the lowest values. SLT measurements at the ONH were influenced by other tissues besides the RNFL like blood vessels and glial tissues. SLT and SLP were most strongly correlated at distances of 1.25 and 1.5 ONHD.

Conclusions: While both imaging technologies are valuable tools in detecting glaucoma, measurements at the ONH rim should be interpreted critically since both methods might provide misleading results. For the assessment of the retinal nerve fiber layer we would like to recommend for both imaging technologies, SLT and SLP, measurements in 1.25 and 1.5 ONHD distance of the rim of the optic nerve head.
\end{abstract}

Keywords: Glaucoma, normal tension glaucoma, retinal nerve fiber layer, scanning laser topography, scanning laser polarimetry, same OD diameter distances.

\section{INTRODUCTION}

Glaucoma, a potentially blinding disease, is characterized by a significant loss of neuronal tissue before the first functional damages and clinical symptoms appear. To initiate treatment before functional loss develops, glaucoma needs to be detected as early as possible. This detection relies on examinations of structural damage to the optic nerve head $(\mathrm{ONH})$ and the retinal nerve fiber layer (RNFL) in combination with an assessment of the visual function, which in the case of glaucoma typically means evaluating the patient's visual field. This assessment of glaucomatous changes depends on the examination method, the patient's compliance, and the ophthalmologist's experience. Therefore, it is prone to subjectivity and variability. The traditional way of documenting these structural changes, stereophotography of the optic disc, requires a subjective assessment as well [1]. Additionally, worldwide it is difficult to obtain a certified device (e.g. in Europe no certified device is available at all).

*Address correspondence to this author at the Eye Hospital, Evangelisches Krankenhaus Gelsenkirchen, Munckelstr. 27, D-45879 Gelsenkirchen, Germany; Tel: 0049 - 209-37261; Fax: 0049-209-378555;

E-mail: stephan.kremmer@arcor.de
The introduction of modern imaging technologies has decreased the dependence on the subjective interpretation of the observer. For the diagnosis of glaucoma, two methods of computer-based scanning laser imaging are widely considered a huge step forward in the early detection and follow-up analysis of glaucomatous changes to the optic nerve head and the retinal ganglion cells: scanning laser topography (SLT) and scanning laser polarimetry (SLP). SLT provides multiple confocal shallow depth-of-field images that are combined to provide a three-dimensional image of the optic disc and its surrounding area - it is, in fact, more a topography than a tomography. SLP measures the thickness of the RNFL based on the change of polarization - called retardation - that occurs when light passes through a birefringent tissue like the microtubules of the retinal nerve fiber layer. In the eye, the RNFL isn't the only tissue with these properties; the cornea [2] and to a much lower extent the crystalline lens [3] are birefringent, too. SLP devices compensate for the effect of this anterior segment birefringence with a special technique; in earlier devices with a fixed corneal compensator (FCC) and since 2002 using variable corneal compensation (VCC) as in current models like the GDx VCC (Carl Zeiss Meditec, Jena, Germany). The latest method, called enhanced corneal 
compensation (ECC), is said to have further improved the evaluation of the RNFL by the SLP device and, according to recent studies [4,5] has increased both the diagnostic accuracy and the correlation between functional and structural measurements. Recent research indicates that there is still a large gap between the diagnostic conclusions reached using these modern imaging devices and the clinical assessment: a study of 312 glaucoma patients and 41 control participants by Pablo et al., [6] found that the agreement between the stereophotographic evaluations and the conclusions based on SLT and SLP was just $52.9 \%$. Medeiros et al., [7] could show already SLP with VCC to be superior to these techniques, and that the new SLP ECC technology again is an advantage in image quality and provides an increased ability to detect early glaucomatous damage and even small changes [8]. It has recently [9] been demonstrated that SLT and SLP are less dependent on scan quality (within the range recommended as acceptable by the manufacturer of each imaging device) than ocular coherence tomography (OCT).

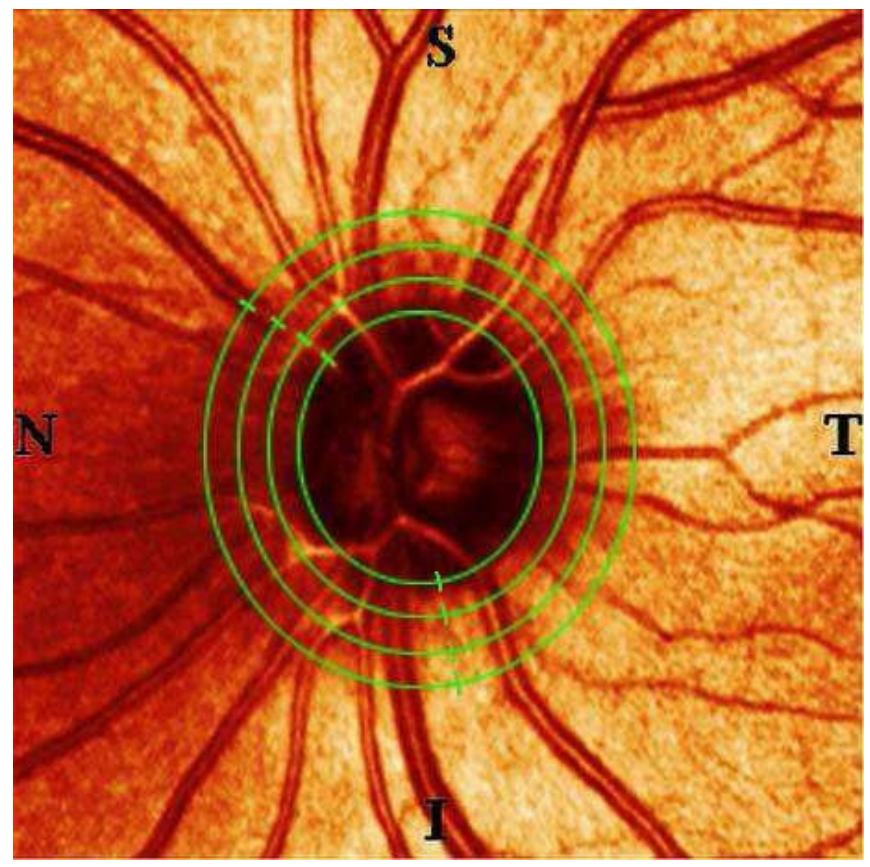

Fig. (1). This picture of a healthy, young individual's optic nerve head shows the four concentric ellipses that were superimposed on each of the participants' SLT and SLP images. The innermost ellipse marks the measurements taken exactly on the nerve head's rim, the other measurements were taken at 1.25 optic nerve head diameters (ONHD), at 1.5 ONHD and at 1.75 ONHD. All data points were saved and documented in the same order: starting on the temporal side (T) and then proceeding towards the superior (S), the nasal $(\mathrm{N})$, the inferior (I) segment und coming full circle back to the temporal periphery.

While there are several similarities between SLT and SLP like the easy handling, the pain- and hassle-free experience for the patient, and the use of scanning laser technology, there are also some fundamental differences in the basic principles of measurement between the two imaging technologies. SLP measures the thickness of a tissue layer with birefringent properties in vivo while SLT provides the ophthalmologist with a three-dimensional image of 32 confocal hight plane profiles. Whenever the observer of these images tries to assess whether obvious changes have occurred which may be interpreted as a sign of glaucomatous damage, it should be kept in mind that SLT does not measure the thickness of the retinal nerve fiber layer directly but rather puts its three-dimensional data in relation to a predetermined reference level. The measurements of both methods correlate with visual field loss in glaucoma patients $[4,8,10,11]$.

In literature, we could only find one comparison of RNFL measurements by means of SLT and SLP [12] revealing significant differences in SLT and SLP measurements but congruences in ratio parameters although SLT measurements were taken at the rim of the optic disc and SLP data at 1.5 OD diameters. But there are important differences of the anatomy at the rim of the optic disc and the area in $1.5 \mathrm{OD}$ diameters, like e.g. a higher density of blood vessels, glial tissues, and the kinking of the nerve fibers downwards to the lamina cribrosa.

Therefore, it seemed worthwhile to us to compare both methods at the same topographical points in clearly defined distances from the optic nerve head to find out

(1) if the layer thickness as measured by SLT and SLP is identical,

(2) if data gathered by both methods does correlate,

(3) if the distance from the optic nerve head rim plays a role, and

(4) if these findings might influence the interpretation of clinical findings by SLT and SLP.

\section{MATERIALS AND METHODOLOGY}

We examined 154 eyes of 96 participants who were assigned to five different groups. 41 eyes (21 individuals) were designated "young normals" (YNorm), 24 eyes (16 individuals) were labelled "older normals" (ONorm), 35 eyes (21 individuals) "normal tension glaucoma" (NTG), 27 eyes (19 individuals) were classified as "open-angle glaucoma with early visual field defect" (OAGE) and 27 eyes (19 individuals) as "open-angle glaucoma with advanced visual field defect" (OAGA) according to the visual field in glaucoma classification of Aulhorn [13]. 4 patients had their right and left eyes assigned to different groups. The average age of the YNorm was 25 years (min. 23 years, max. 27 years), the average age of the ONorm was 64.8 years (min. 58 years, max. 83 years), in the NTG group it was 67.3 years (min. 46 years, max. 81 years), in the OAGE group 60.8 years (min. 37 years, max. 82 years) and in the OAGA group 64.7 years (min. 37 years, max. 82 years). 52 of our study objects were male, 44 were female. The young healthy subjects were students at the University of Essen, all individuals in the 4 other groups were patients at the University Eye Hospital Essen.

The ophthalmological evaluation all these individuals underwent consisted of

determining visual acuity and refractive error,

slit-lamp examination,

tonometry (applanation) and, in glaucoma patients, a 24-hour profile of the intraocular pressure (IOP), 
- $\quad$ static perimetry (Twinfield Automatic Perimetry, Oculus, Wetzlar, Germany),

- SLP,

- $\quad$ SLT, and

- ophthalmoscopic examination of the posterior segment of the eye.

For scanning laser polarimetry we used a GDx/NFA, version 2.010 E beta (LDT, San Diego, California, currently Carl Zeiss Meditec, Jena, Germany) which is based on an aluminium-arsenic laser (wavelength $780 \mathrm{~nm}$, maximal retinal illumination $0.025 \mathrm{Watt} / \mathrm{cm}^{2}$ ). Scanning laser topography was performed with a TopSS, software version 3.0.19 (LDT, San Diego, California currently Carl Zeiss Meditec, Jena, Germany), a device that uses a galliumaluminum-arsenide laser (wavelength $780 \mathrm{~nm}$, maximal retinal illumination $0,025 \mathrm{Watt} / \mathrm{cm}^{2}$ ) as reported elsewhere [14]. To draw a valid comparison between the two imaging technologies, using images of exactly the same size depicting exactly the same area in identical resolution was essential. All images used for this study were centered on the optic nerve head, their size was $15^{\circ}$ by $15^{\circ}$ and they had a resolution of $256 \times 256$ pixels.

In the first step, three images were taken with the SLT device and a mean image was calculated. The same was done with the SLP instrument. These mean images were used for the further procedures. The mean image of the SLT device, containing 65,536 height data points, was exported to a file and stored on optical discs. Deriving from the TopSS technique in many points, e.g. the same data base structure was used in all TopSS and GDx/NFA instruments. Therefore, the GDx/NFA software was able to import the TopSS data and afterwards to read both kinds of data, the topographic height information of the SLT and the polarimetric data of the GDx as well. Even the implemented alignment software was identical and could be employed for measurements of both instruments.

For the following evaluation steps, the SLP computer was used. Four concentric ellipses were superimposed on each of the mean images: the first one measuring exactly at the rim of the optic nerve head (1.0 ONHD), the next measuring at 1.25 optic nerve head diameters (ONHD), the third at $1.5 \mathrm{ONHD}$ and the fourth measuring at 1.75 ONHD. Each circle was 5 pixels wide (Fig. 1). Since SLT measures the height of the scanned anatomical structures relative to a reference level, some of its data can be negative. To establish a system that would convert all registered data points into positive numbers, we defined the lowest point in each examined eye as zero with the remaining data points determined by their elevation relative to this zero level. The data gained using SLP was treated the same way.

All data points were saved and documented in the same order: temporal superior, superior, nasal, inferior and temporal inferior (TSNIT).

All statistical analyses (Wilcoxon test, Spearman correlation) were calculated using SPSS statistical software for Windows.

\section{RESULTS}

Presented here are the curves and their characteristics for the 5 different groups. The figures show from top to bottom the measured elevation of the RNFL in each group of individuals at the rim of the $\mathrm{ONH}$ (1.0 ONHD), at 1.25 ONHD, at 1.5 ONHD and at 1.75 ONHD, respectively.

\section{Young, Normal Individuals (YNorm)}

For both imaging technologies, the rule for most groups is a twin peak-shaped curve with maxima in both the superior and the inferior sector at the rim of the optic nerve head (1.0 ONHD). This is much more distinct in SLT than in SLP. The two peaks of the SLT lose their prominence with increasing distance of the data points from the optic nerve head. In healthy young individuals, SLT has its peaks at 437 $\mu \mathrm{m}$, i.e. in the superior quadrant. This highest level is reduced by $102 \mu \mathrm{m}$ at 1.25 ONHD, decreases by a further 73 $\mu \mathrm{m}$ at $1.5 \mu \mathrm{m}$ ONHD and measures $230 \mu \mathrm{m}$ at 1.75 ONHD while still being the highest elevation of the SLT curve. The peak in the inferior sector of the optic nerve head is of 399 $\mu \mathrm{m}$ height at $1 \mathrm{ONHD}$ and decreases to just below $150 \mu \mathrm{m}$ at 1.75 ONHD.

The superior peak shows an overall decrease in height (from the innermost to the outermost circle) of $52.6 \%$, the peak in the inferior sector decreases by $36.34 \%$. The SLP has peaks in the superior and inferior sector as well, but their maxima increase from 1.0 ONHD $(61 \mu \mathrm{m}$ for the highest point of the superior sector) through 1.75 ONHD $(77 \mu \mathrm{m}$ for the superior peak). In general, the largest increase of nerve fiber layer thickness is between the measurement ellipses at 1.0 and 1.25 ONHD (Fig. 2).

\section{Older Normal Individuals (ONorm)}

Unlike in YNorm, the highest peak of SLT measurements in older healthy (i.e. non-glaucomatous) individuals at 1.0 ONHD is in the inferior, not in the superior sector. At the other points of measurement, the inferior peak is slightly below the highest level of the superior segment. The superior peak decreases from 1.0 ONHD through 1.75 ONHD by $46 \%$, the inferior peak by $57 \%$.

The SLP curve shows a slight elevation representing a thickening of the RNFL from 1.0 through 1.25 ONHD. The data remain relatively consistent at the outermost measurement zones (Fig. 3).

\section{Patients with Normal Tension Glaucoma (NTG)}

Once again, in SLT the peak at 1.0 ONHD is in the inferior sector, while at the other distances from the optic nerve head's rim the height maxima are located in the superior quadrant. This peak decreases from 1.0 ONHD through 1.75 OHND by $49 \%$. The SLP indicates an increasing RNFL thickness from 1.0 through 1.25 up to 1.5 ONHD in both the superior and the inferior quadrants. At 1.75 ONHD, however, there is a slight reduction of that layer's thickness. The superior quadrant's height increases by $27 \%$, the inferior quadrant's height by $37 \%$ (Fig. 4). 


\section{Young, normal individuals (YNorm)}

a

$1.0 \mathrm{ONHD}$

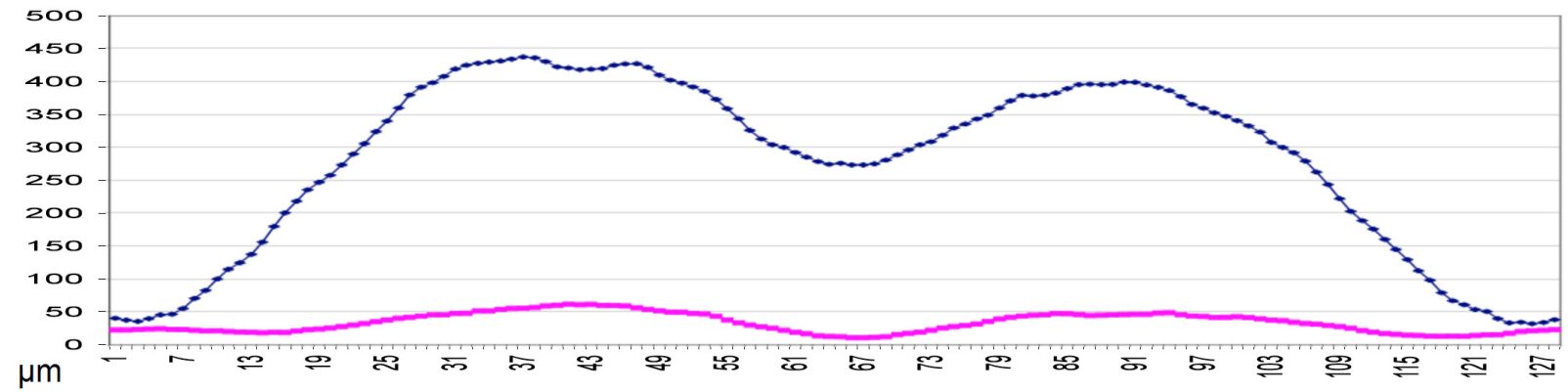

b

$1.25 \mathrm{ONHD}$

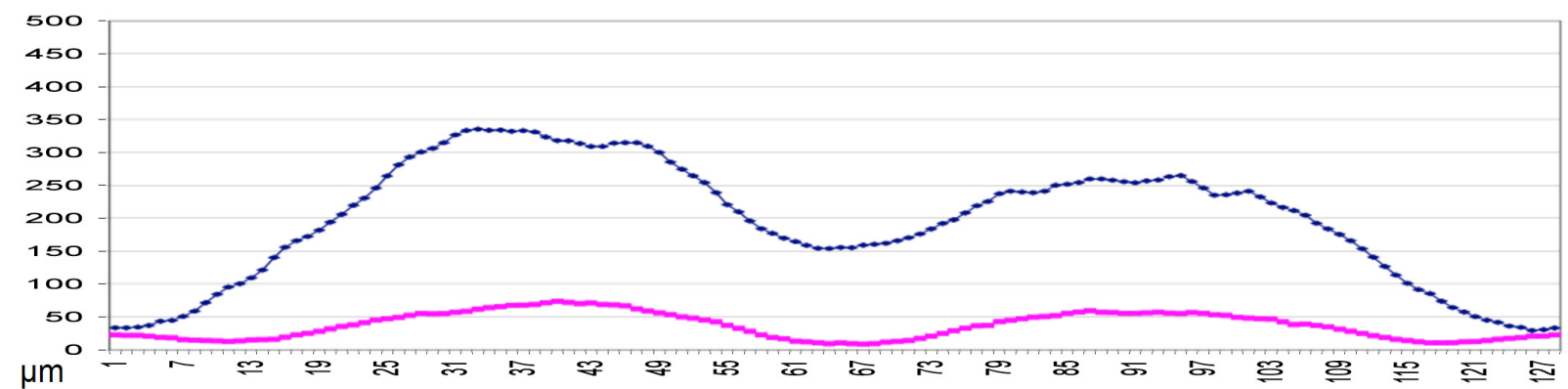

C

$1.5 \mathrm{ONHD}$

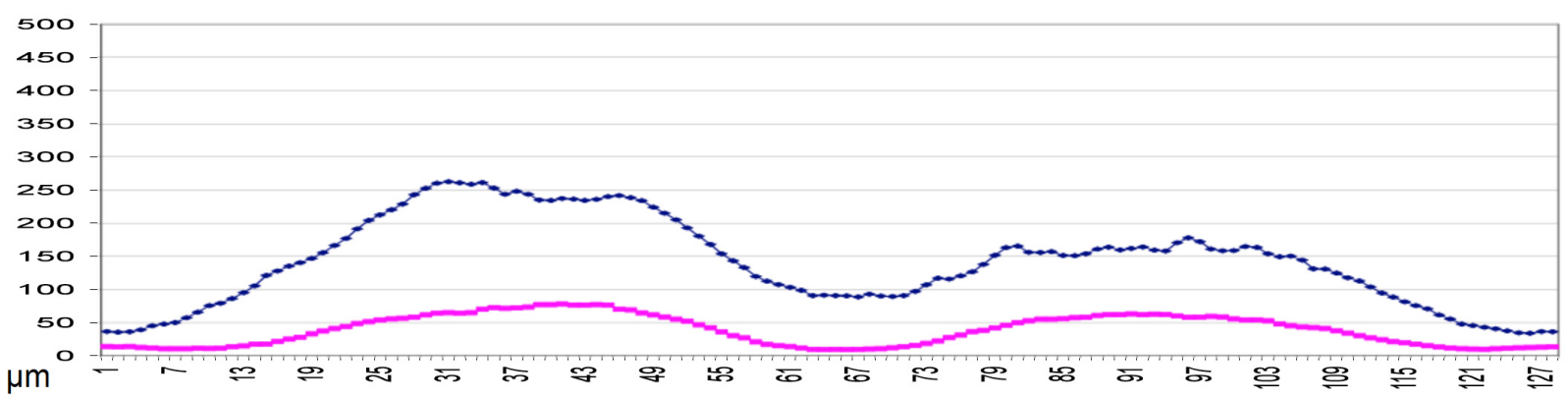

d

1.75 ONHD

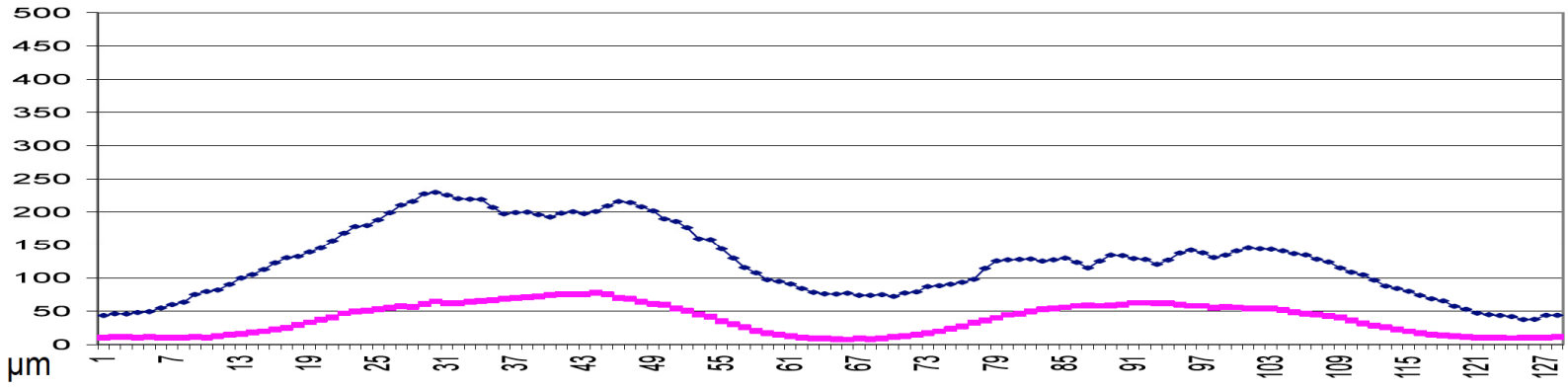




\section{Older, normal individuals (ONorm)}

(Figs. 2-6) contd.....

a

$1.0 \mathrm{ONHD}$

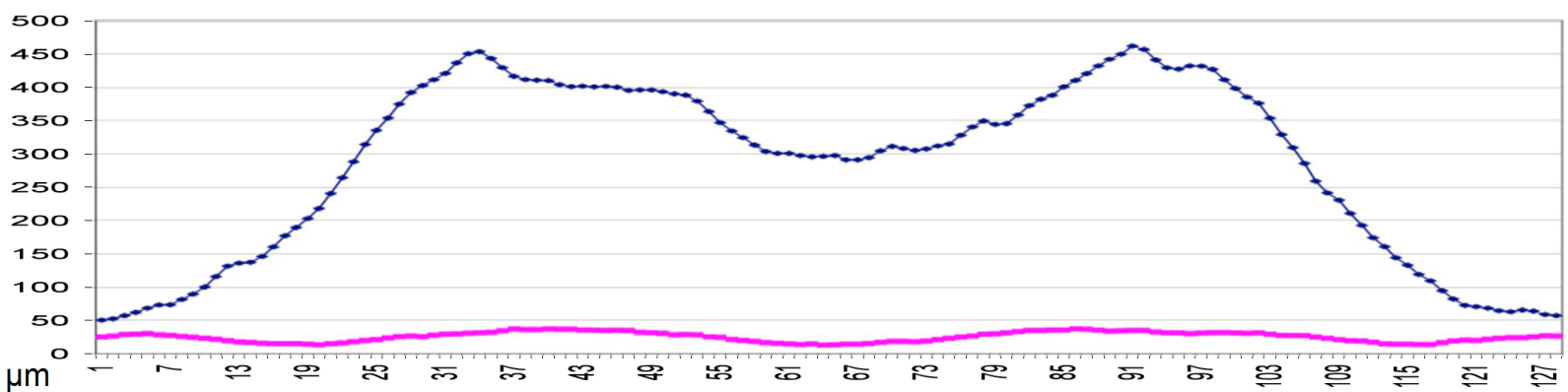

b

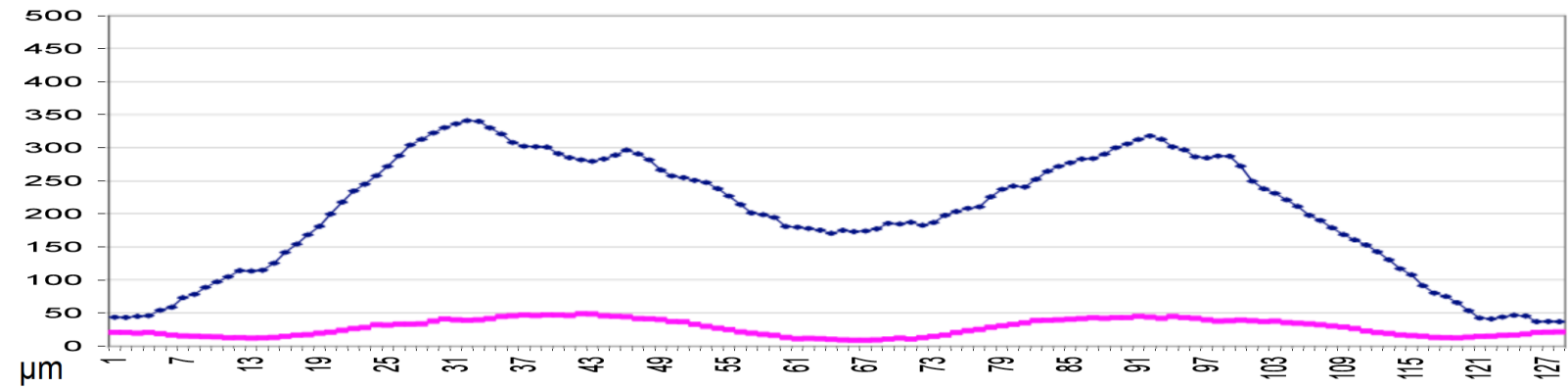

C

1.5 ONHD

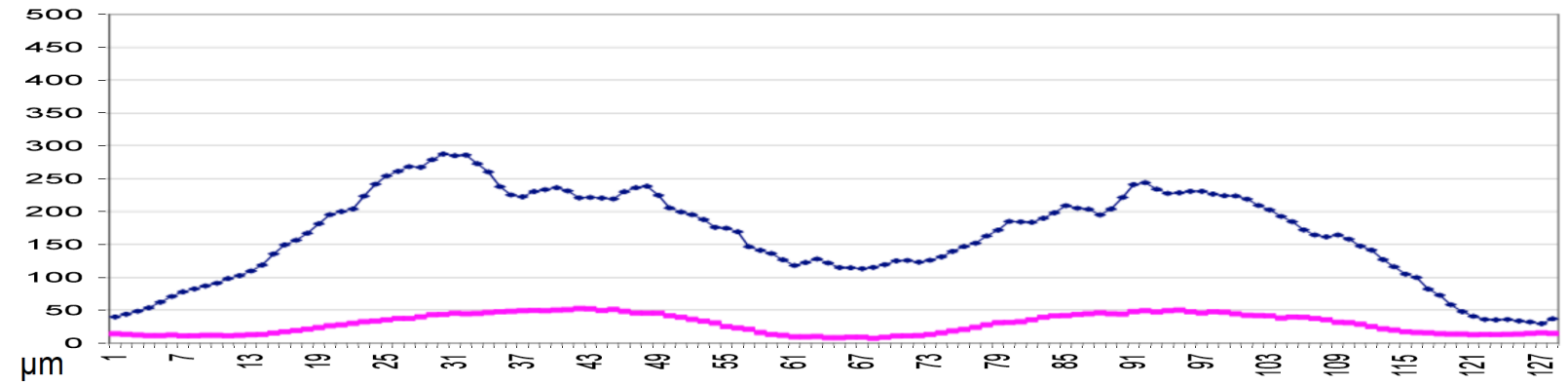

d

1.75 ONHD

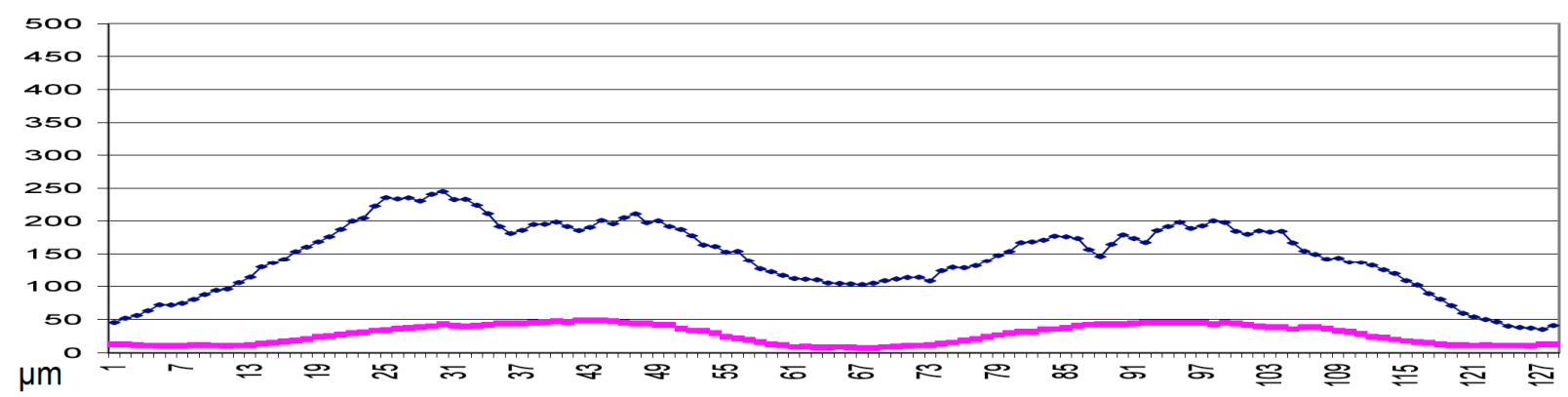


(Figs. 2-6) contd.....

\section{Normal tension glaucoma (NTG)}

a

$1.0 \mathrm{ONHD}$

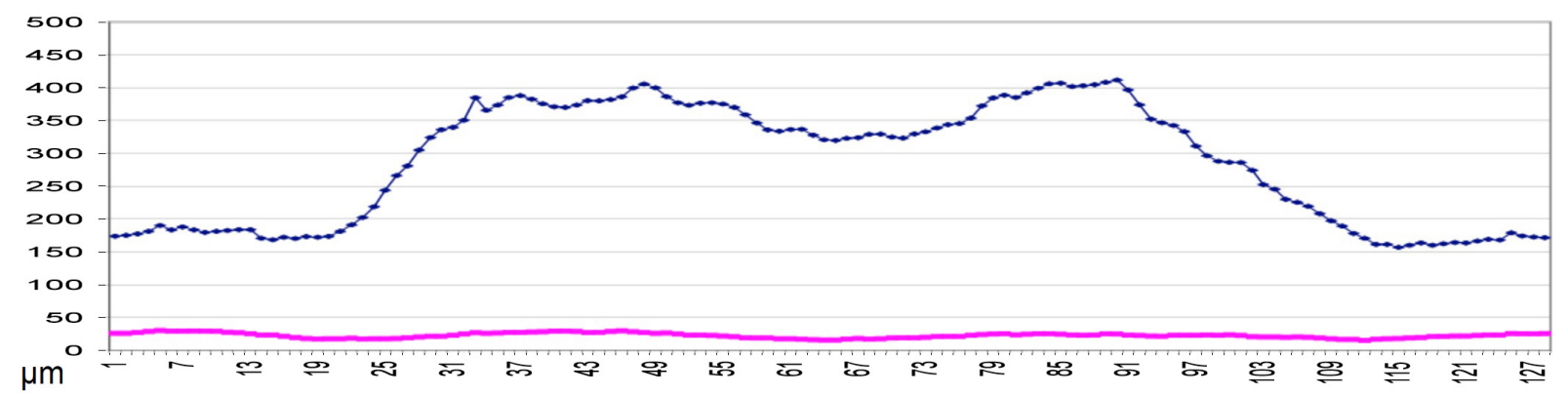

b

$1.25 \mathrm{ONHD}$

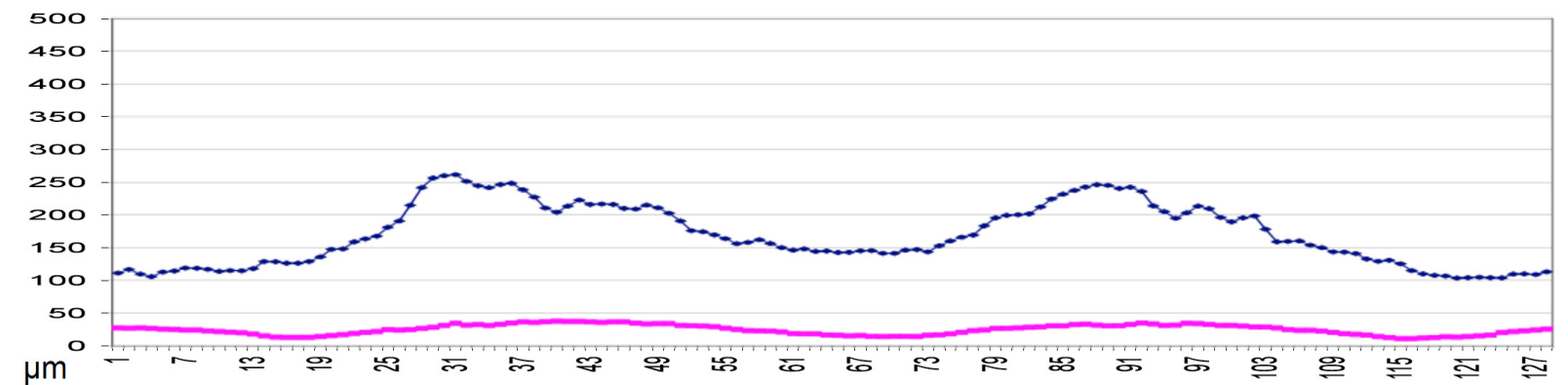

C

$1.5 \mathrm{ONHD}$

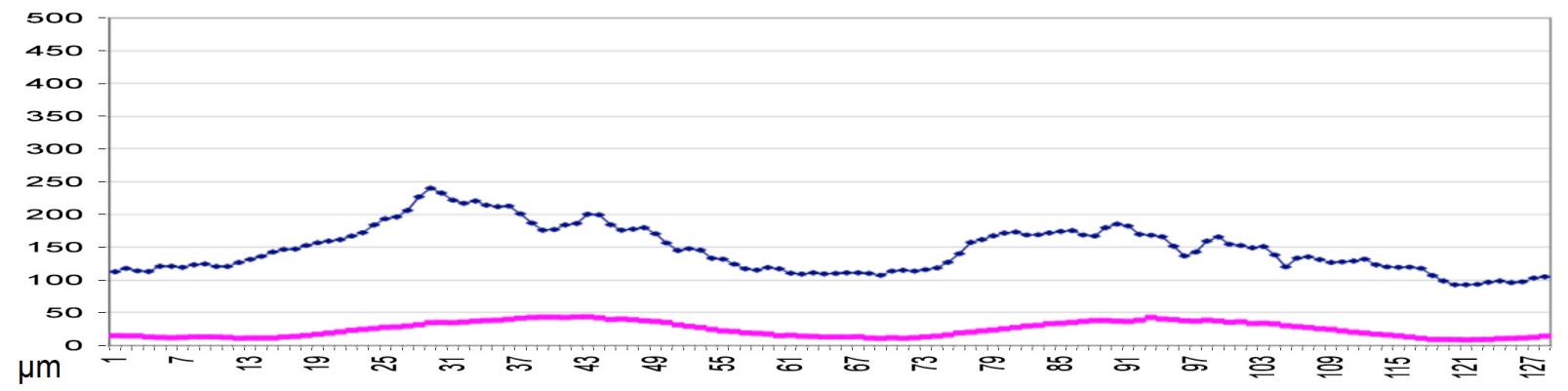

d

$1.75 \mathrm{ONHD}$

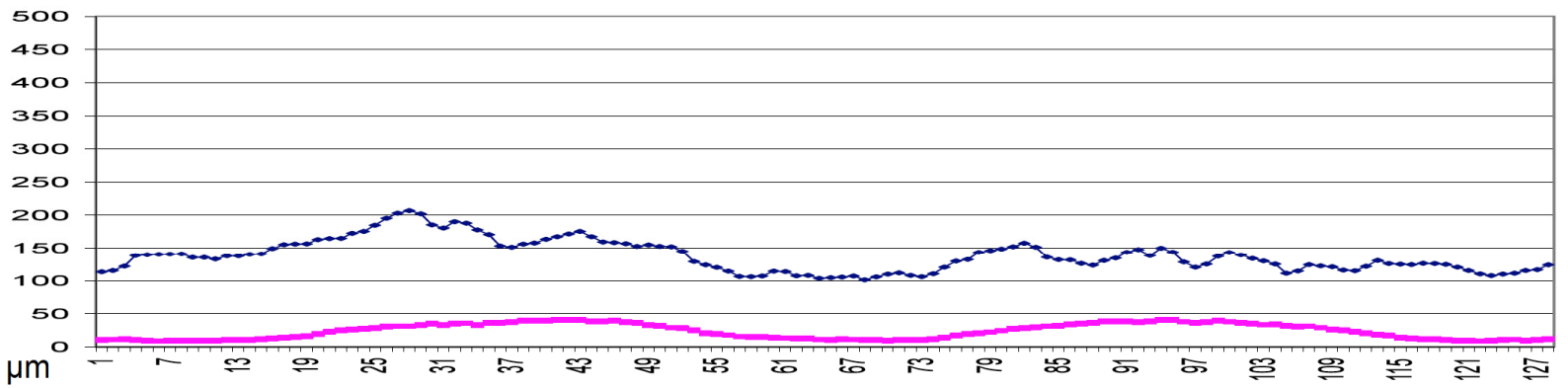




\section{Glaucoma, early VF defects (OAGE)}

(Figs. 2-6) contd.....

a

$1.0 \mathrm{ONHD}$

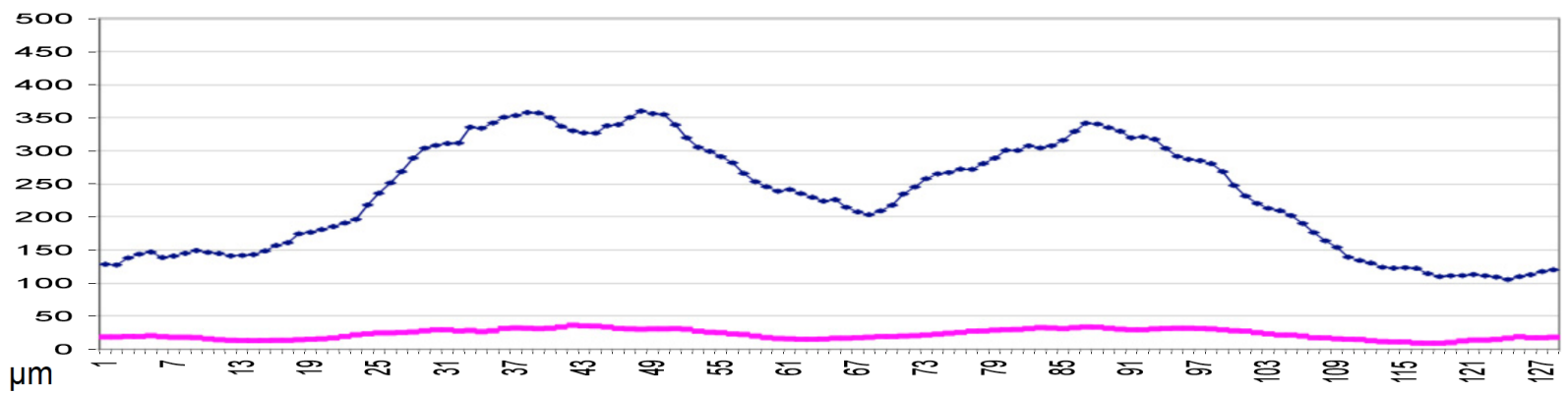

b

1.25 ONHD

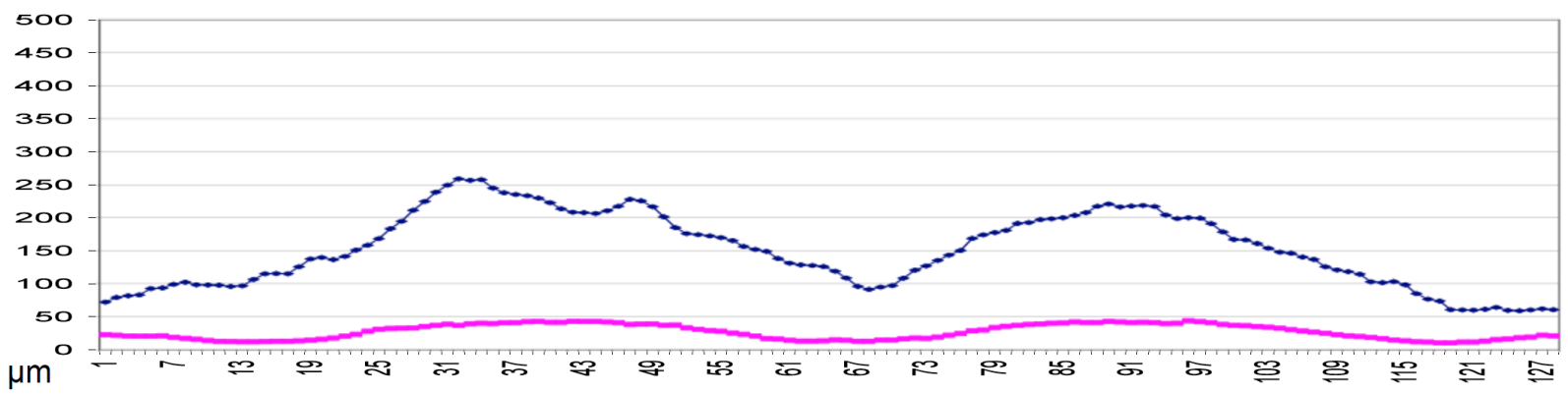

C

1.5 ONHD

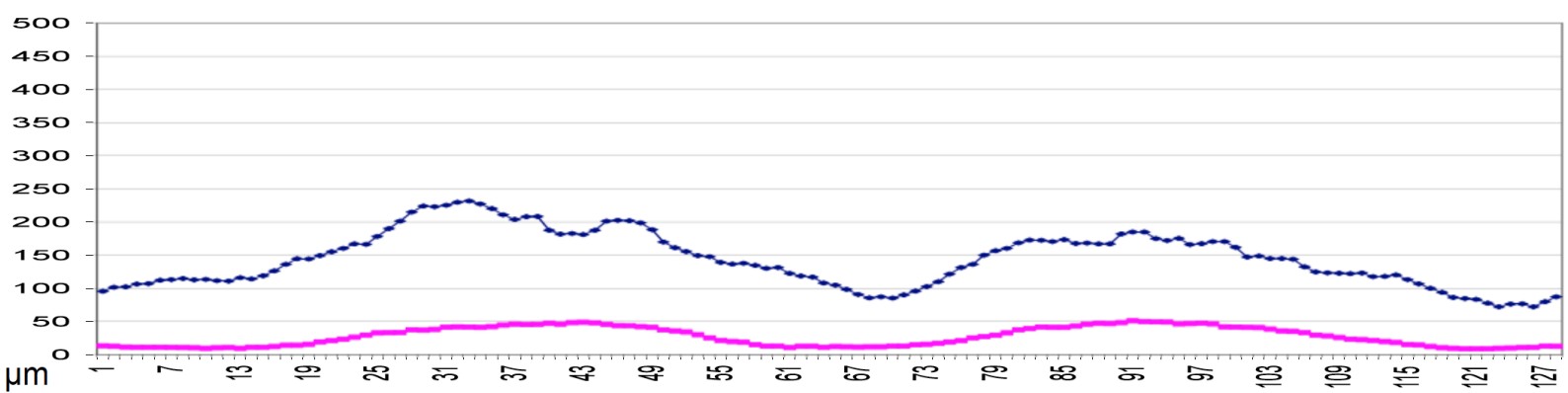

d

1.75 ONHD

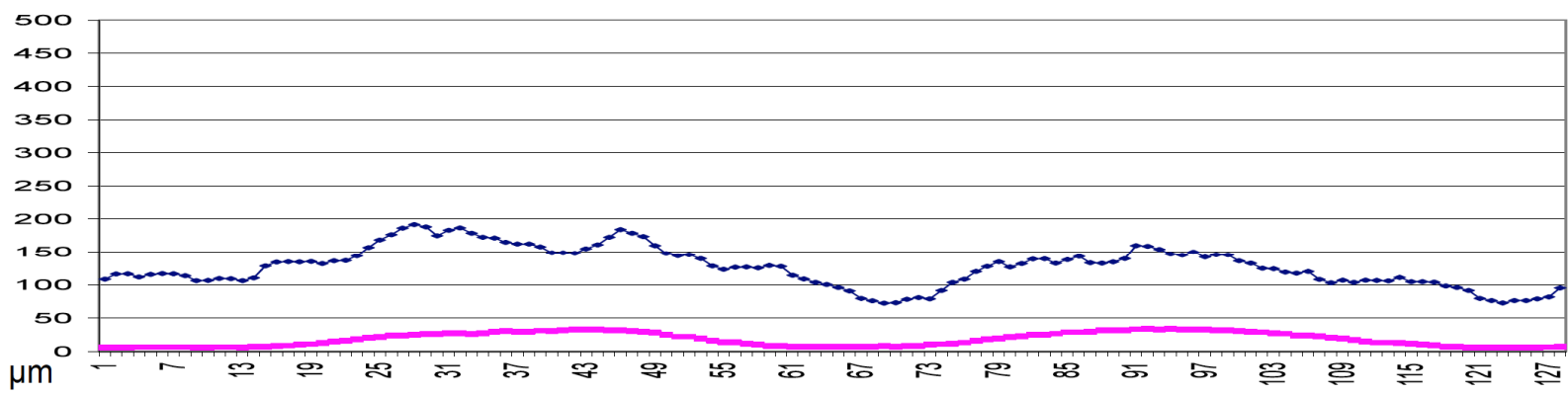


(Figs. 2-6) contd.....

\section{Glaucoma, advanced VF defect (OAGA)}

a

$1.0 \mathrm{ONHD}$

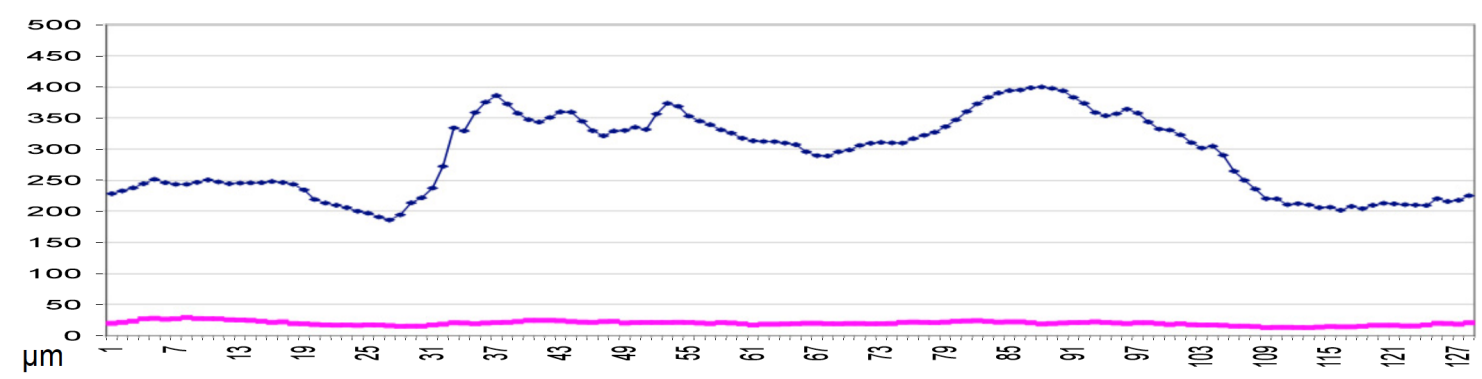

b

1.25 ONHD

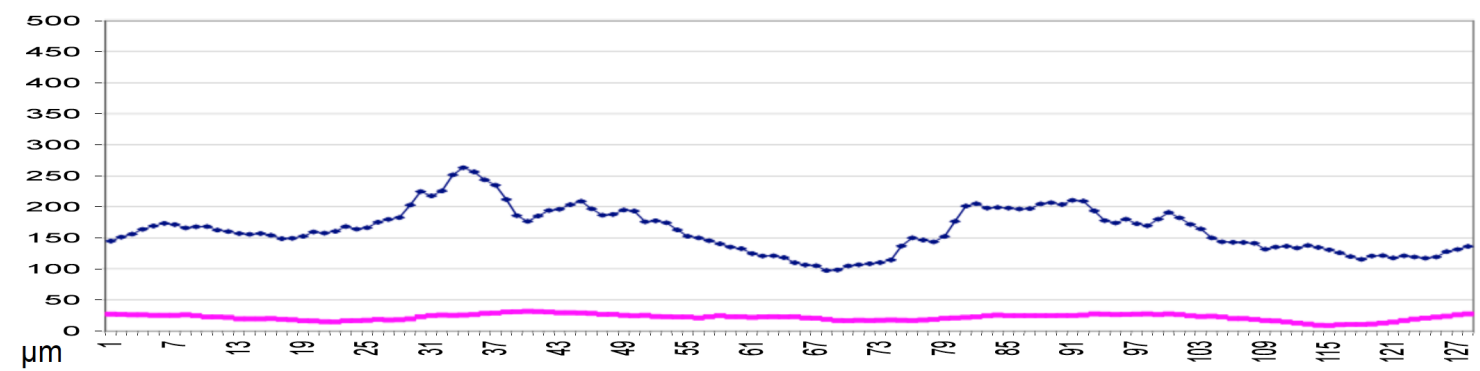

C

$1.5 \mathrm{ONHD}$

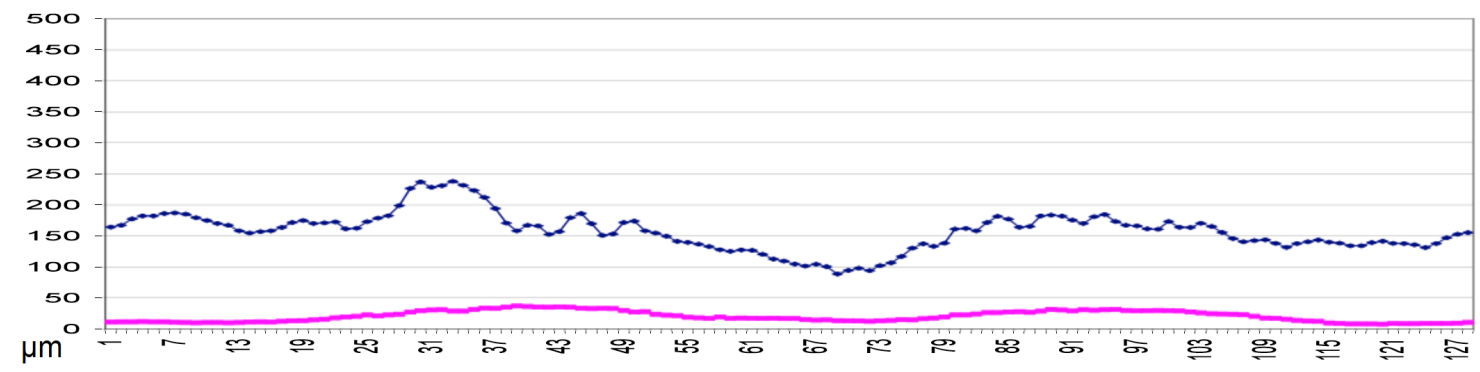

d

1.75 ONHD

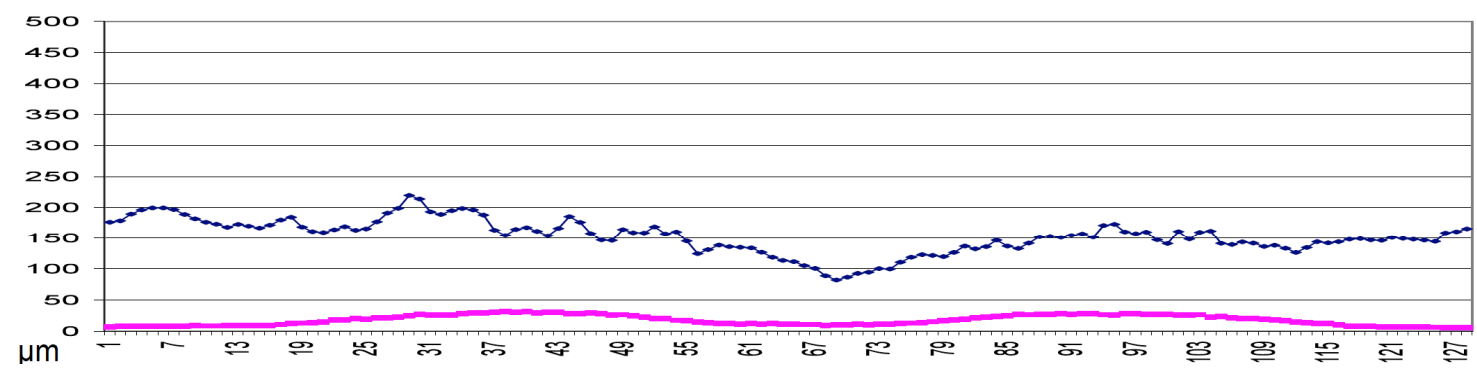

Figs. (2-6). The curves showing the retinal thickness of young, normal individuals (YNorm), of older, normal individuals (ONorm), of patients with normal tension glaucoma (NTG), of open-angle glaucoma patients with early visual field defects (OAGE) and of open-angle glaucoma patients with advanced visual field defects (OAGA) as measured using an SLT device (blue) and an SLP device (red). Measurements taken from top to bottom at the rim of the optic nerve head and in increasing distances as shown in Fig. (1). The numbers 1-16 on the $\mathrm{x}$-axis mark the temporal superior sector, 17-48 the superior, 49-80 the nasal, 81-112 the inferior, 113-128 the temporal inferior sectors, respectively. 


\section{Patients with Open-Angle Glaucoma and Early Visual Field Defect (OAGE)}

The SLT peak in the superior sectors seems to be split in patients with early glaucomatous damage: there are two similar peaks at 1.0 ONHD, each having an elevation of about $360 \mu \mathrm{m}$. The inferior peak's maximum height at 1.0 ONHD is $259 \mu \mathrm{m}$. It decreases towards the periphery by $53 \%$.

The superior peak at 1.75 ONHD is, compared to the twin elevations at 1.0 ONHD, lower by $47 \%$. SLP has a maximum height at $1.0 \mathrm{ONHD}$ of $36 \mu \mathrm{m}$ for the superior and of $34 \mu \mathrm{m}$ for the inferior quadrant right at the rim of the optic disc. The RNFL gets thicker at the next two measurement circles with the inferior segment being the thickest. At $1.75 \mu \mathrm{m}$ there is a marked decrease with a maximum height of $33 \mu \mathrm{m}$ for the superior and of $34 \mu \mathrm{m}$ for the inferior peak. This represents a sharp decrease of the RNFL thickness both superiorly and inferiorly compared to the height at 1.5 ONHD by about a third (Fig. 5).

\section{Patients with Open-Angle Glaucoma and Advanced Visual Field Defect (OAGA)}

The SLT maxima at the rim are $400 \mu \mathrm{m}$ in the inferior and $386 \mu \mathrm{m}$ in the superior sector. At the next circle $(1.25$ ONHD) there already is a sharp decline of $189 \mu \mathrm{m}(47 \%)$ and $123 \mu \mathrm{m}(32 \%)$, respectively. The further decrease is less distinct: to $238 \mu \mathrm{m}$ and $219 \mu \mathrm{m}$ for the superior peak and to $184 \mu \mathrm{m}$ and $173 \mu \mathrm{m}$ for the inferior peak, at distances of 1.5 and 1.75 ONHD, respectively. The SLP curve at 1.0 ONHD seems almost flat, with a difference of only $16 \mu \mathrm{m}$ between the highest and the lowest point. The maximum $(29 \mu \mathrm{m})$ is in the temporal quadrant while at $1.25 \mathrm{ONHD}$ the highest point $(32 \mu \mathrm{m})$ is in the superior sector. At 1.5 and 1.75 ONHD the highest elevation is found in the superior $(37 \mu \mathrm{m}$ at 1.5 ONHD and $32 \mu \mathrm{m}$ at $1.75 \mathrm{ONHD}$ ) and in the inferior sector (31 $\mu \mathrm{m}$ and $28 \mu \mathrm{m}$ ), respectively (Fig. 6).

\section{DISCUSSION}

In our comparison of SLT and SLP measurements, there is a general tendency for the SLT values to decrease from the rim of the optic disc towards the periphery while SLP values tend to increase with distance from the optic nerve head. Younger and older healthy subjects had similar SLT curves with increasing elevation from 1.0 through 1.5 and a slight decline at 1.75 OHND. Glaucoma patients had quite different curves with an irregular pattern of height reduction from the rim of the nerve head towards the periphery. The largest decrease of nerve layer height occurred between 1.0 and 1.25 ONHD. All three groups of glaucoma patients showed only a minor decrease in thickness from 1.25 to 1.5 ONHD, with an almost flat curve (= no decrease) in glaucoma patients with advanced damage. The decrease from 1.5 to 1.75 ONHD was similar in all three patient groups. In SLP curves, there was a more pronounced drop in thickness from 1.5 to 1.75 in patients with early glaucomatous damage. In advanced glaucoma, values showed a low increase and a low modulation of the TSNIT curves.

The peaks in the superior and the inferior segments of the optic nerve head, visible in SLT as well as in SLP, are consistent with the optic disc's anatomy: in these segments there is a strong accumulation of nerve fibers entering the optic nerve head [15]. Histological studies in combination with the use of imaging technologies [16], have demonstrated that the RNFL is strongest in the inferior segment, just slightly thinner in the superior quadrant with decreasing thickness in the nasal, and, finally, the temporal segment. Klemm [15] as well as Jonas and Dichtl [17] have demonstrated that the nerve fiber layer increases in thickness from the periphery towards the optic nerve head.

Furthermore, the measurements reflect physiological and pathological changes: young healthy individuals have a thick RNFL, in elder normal participants the RNFL gets thinner due to the age-related loss of retinal neurons. Glaucoma patients have reduced curve modulations with reduced increases of the inferior and superior nerve fiber layer indicating the demise of retinal ganglion cells, the crucial pathophysiological event so typical for this disease. Since SLT registers changes in height relative to a level of reference and not a specific decrease or increase in the thickness of the RNFL, the hight values measured at 1.0 ONHD which are not in accordance with the histology of the layer at the rim seem to indicate the influence of another tissue: in this part of the optic nerve head the vessels are lying close together and have relatively thick diameters. Therefore, SLT measurements in this region will lead to results which are considerably higher than in the periphery [18]. Glia cells contribute to a relative increase in height at the neuroretinal rim and may further influence the measurements [19], especially in glaucoma [20].

SLP tends to measure the lowest values at the rim of the optic nerve head (1.0 ONHD). Here the axons of the retinal ganglion cells unite and continue in a funnel-shaped bundle kinking downwards to the lamina cribrosa. This causes a change in the laser beam's angle of incidence. But if the laser beam is not perpendicular, it passes less through the tissues but is rather following the kinking nerve fibers. It hits fewer optical boundaries, the phase shift introduced by the birefringent material and consequently the retardation values are lower, and the SLP device calculates a thinner nerve fiber layer thickness.

It is well established that data gathered by SLP technology from the rim of the optic nerve head differs considerably from measurements taken more peripherally. Therefore, even in the beginning of the clinical use of SLP it has been suggested by Glück et al., [21] to omit results of SLP evaluations performed at the rim of the ONH.

The same anatomical circumstances affect SLT, too, but with increasing instead of decreasing values. The superior and inferior veins and arteries do not only influence the RNFL profile using SLT but also the results of another technology, optical coherence tomography (OCT) as demonstrated by Hood et al., [22] and Medeiros et al. [8]. Basically, the OCT technique is able to measure blood vessels but unfortunately, many of the present software releases are not using this information for their clinical patient reports [23]. Hopefully, new OCT software versions will exclude vessels from nerve fiber measurements as well as already provided by SLP today. Further towards the periphery, medium and large scan circles around the optic nerve head have just recently been proven useful for RNFL evaluation in glaucoma by Dada et al. [24]. 
SLT and SLP were most strongly correlated at distances of 1.25 and 1.5 ONHD from the rim of the optic nerve head. Both technologies are reliable in detecting relative changes of the retinal nerve fiber layer. The absolute values provided by these instruments, however, have to be treated cautiously. Different to OCT tomography, SLT measures height values of the retinal surface but it cannot detect what kind of tissue is under the surface. Therefore, SLT provides information of the sum of all tissues under the surface, e.g. the RNFL but also blood vessels and glial tissue. In addition, SLT measurements depend on a 'reference level' which is prone to intra- and interindividual variations and is thus difficult to define. It is undoubtedly a huge advantage of SLP, as stated by Hoffmann [25], that this technology works without drawing a contour line and a reference plane. In a long-term follow-up study, Funk et al., [11] reported a similar diagnostic value of SLT and perimetry in glaucoma patients. While follow-ups using SLP hitherto are limited, SLP has shown its value as a screening tool for glaucoma and, using a combination of different criteria, outperformed SLT for this purpose $[5,7,10]$. With the use of the new ECC technology trend and event analyses are possible, and the image quality even improved especially in patients with atypical nerve fiber layer patterns $[4,8,10]$.

While SLT and SLP evaluations might look similar at first glance, there are considerable differences between the measurements both methods provide, in particular at the rim of the optic nerve head and in cases of advanced glaucoma. Therefore, a comparison of SLT (HRT) measurements at the rim of the optic disc with SLP (GDx) data at $1.5 \mathrm{OD}$ diameters [12] should be seen with the necessary caution. In the present study we did not use an HRT but a Topographic Scanning System (TopSS) on one hand because the technique is very similar. Both instruments derive from the former Laser Tomographic Scanner, Heidelberg Instruments before the split into Heidelberg Engineering (HRT) and Laser Diagnostic Technologies (TopSS and GDx/NFA) took place. For our purposes the advantage of the TopSS was that it had exactly the same data base structure like the GDx/NFA, enabling to export and afterwards to read the topographical and polarimetrical data with the same instrument. Even the implemented alignment software of TopSS and GDx/NFA was identical and worked properly with data from both devices allowing for the first time an exact 'pixel by pixel' comparison of data from both technologies, SL Topography and SL Polarimetry. In more actual instrument versions the data base technologies developed differently and unfortunately a direct comparison of data is not possible anymore.

\section{CONCLUSIONS}

Both imaging technologies are valuable tools in detecting glaucoma. But measurements at the $\mathrm{ONH}$ rim should be interpreted critically since both methods might provide misleading results. Here, the SLP device may calculate a thinner nerve fiber layer thickness because the axons of the retinal ganglion cells begin to kink downwards to the lamina cribrosa, and the laser beam may be not perpendicular.

SLT provides information of the sum of all tissues under the surface, e.g. the RNFL but also blood vessels and glial tissue. In addition, SLT measurements depend on a 'reference level' which is prone to intra- and interindividual variations and is thus difficult to define. The observer should cross-check data gained from the rim with the clinical features and ponder the measurement's plausibility. For the assessment of the retinal nerve fiber layer we would like to recommend for both imaging technologies, SLT and SLP, measurements in 1.25 and 1.5 ONHD distance of the rim of the optic nerve head.

\section{ACKNOWLEDGEMENT}

Supported by Alfried Krupp von Bohlen und Halbach Foundation, Essen, Germany.

\section{CONFLICTS OF INTEREST}

None declared.

\section{REFERENCES}

[1] Bowd C, Zangwill L, Bourne R, Weinreb R. Scanning laser imaging. In: Choplin N, Lundy D, Eds. Atlas of glaucoma. Boca Raton, Florida: Informa Healthcare 2007; pp. 75-87.

[2] Greenfield DS, Knighton RW, Feuer W, Schiffman J, Zangwill L, Weinreb RN. Correction for corneal polarization axis improves the discriminating power of scanning laser polarimetry. Am J Ophthalmol 2008; 134: 27-33.

[3] Kremmer S, Garway-Heath DF, de Cilla S, Steuhl K-P, Selbach JM. Influence of cataract surgery with implantation of different intraocular lenses on scanning laser tomography and polarimetry. Am J Ophthalmol 2003; 136: 1016-21.

[4] Lemij H, Reus N. New developments in scanning laser polarimetry for glaucoma. Curr Opin Ophthalmol 2008; 19: 136-40.

[5] Tóth M, Holló G. Increased Long-term measurement variability with scanning laser polarimetry employing enhanced corneal compensation: an early sign of glaucoma progression. J Glaucoma 2008; 17: 571-7.

[6] Pablo LE, Larrosa J, Polo V, Ferreras A, Alias E, Honrubia F. Performance of GDx and HRT in the Finnish Evidence Based Guideline for open-angle glaucoma. Eye 2009; 24: 297-303.

[7] Medeiros F, Vizzeri G, Zangwill L, Alencar L, Sample P, Weinreb $\mathrm{R}$. Comparison of retinal nerve fiber layer and optic disc imaging for diagnosing glaucoma in patients suspected of having the disease. Ophthalmology 2008; 115: 1340-6.

[8] Medeiros F, Zangwill L, Alencar L, Sample P, Weinreb R. Rates of progressive retinal nerve fiber layer loss in glaucoma measured by scanning laser polarimetry. Am J Ophthalmol 2010; 149: 908-15.

[9] Sung KR, Wollstein G, Schuman JS, et al. Scan quality effect on glaucoma discrimination by glaucoma imaging devices. $\mathrm{Br} \mathrm{J}$ Ophthalmol 2009; 93: 1580-4.

[10] Tóth M, Kòthy P, Holló G. Accuracy of scanning laser polarimetry, scanning laser tomography, and their combination in a glaucoma screening trial. J Glaucoma 2008; 17: 639-46.

[11] Funk J, Mueller H. Comparison of long-term fluctuations: laser scanning tomography versus automated perimetry. Graefes Arch Clin Exp Ophthalmol 2003; 241: 721-4.

[12] Iester M, Mermoud A. Retinal nerve fiber layer measured by Heidelberg retina tomograph and nerve fiber analyzer. Eur J Ophthalmol 2005; 15: 246-54.

[13] Aulhorn E, Karmeyer H. Frequency distribution in early glaucomatous visual field defects. Doc Ophthalmol 1977; 14: 7583.

[14] Kremmer S, Ayertey H, Selbach JM, Steuhl KP. Scanning laser polarimetry, retinal nerve fiber layer photography, and perimetry in the diagnosis of glaucomatous nerve fiber defects. Graefes Arch Clin Exp Ophthalmol 2000; 238: 922-6.

[15] Klemm M, Rumberger E, Walter A, Richard G. Quantifizierung der retinalen Nervenfaserschichtdicke. Ophthalmologe 2001; 98: 832-43.

[16] Miglior S. GDx in glaucoma. Acta Ophthalmol Scand 2002; 80: 36-40.

[17] Jonas J, Dichtl A. Evaluation of the retinal nerve fiber layer. Surv Ophthalmol 1996; 40: 369-78. 
[18] Dichtl A, Jonas J, Mardin C. Comparison between tomographic scanning and photographic measurement of the neuroretinal rim. Am J Ophthalmol 1996; 121: 494-501.

[19] Weinreb R, Shakiba S, Sample P, et al. Association between quantitative nerve fiber layer measurement and visual field loss in glaucoma. Am J Ophthalmol 1995; 120: 732-8.

[20] Pena JD, Agapova O, Gabelt BT, et al. Increased elastin expression in astrocytes of the lamina cribrosa in response to elevated intraocular pressure. Invest Ophthalmol Vis Sci 2001; 42: 2303-14.

[21] Glück R, Rohrschneider K, Kruse FE, Völcker HE. Nachweis von glaukomatösen Nervenfaserschäden. Ophthalmologe 1997; 94: $815-20$.

[22] Hood DC, Salant JA, Arthur S, Rich R, Liebmann J. The location of the inferior and superior temporal blood vessels and interindivi- dual variability of the retinal nerve fiber layer thickness. J Glaucoma 2010; 19: 158-66.

[23] Kremmer S. Nervenfaserdiagnostik - Fallbeispiele. In: Krieglstein GK, Ed. Glaukom und Makula. Heidelberg: Springer Medizin Verlag 2011; pp. 15-58.

[24] Dada T, Gadia R, Aggraval A, Dave V, Gupta V, Sihota R. Retinal nerve fiber layer thickness measurement by scanning laser polarimetry $(\mathrm{GDxVCC})$ at conventional and modified diameter scans in normals, glaucoma suspects, and early glaucoma patients. J Glaucoma 2009; 18: 448-52.

[25] Hoffmann E, Schulze A. Glaucoma diagnosis using scanning laser polarimetry. Ophthalmologe 2009; 106(8): 696-8, 700-1.

(C) Kremmer et al.; Licensee Bentham Open.

This is an open access article licensed under the terms of the Creative Commons Attribution Non-Commercial License (http: //creativecommons.org/licenses/by$\mathrm{nc} / 3.0 /$ ) which permits unrestricted, non-commercial use, distribution and reproduction in any medium, provided the work is properly cited. 